\title{
Analyse der Störfestigkeit nichtlinearer Schaltungen gegenüber leitungsgebundenen elektromagnetischen Störungen mit multi-input Wiener/Hammerstein-Modellen
}

\author{
S. Stegemann, W. John, and W. Mathis \\ Institut für theoretische Elektrotechnik, Leibniz Universität Hannover, Appelstraße 9A, 30167 Hannover, Deutschland \\ Correspondence to: S. Stegemann (stegemann@tet.uni-hannover.de)
}

\begin{abstract}
Zusammenfassung. In dieser Arbeit wird der Einfluss von leitungsgebundenen Störungen untersucht, welche in einen beliebigen Eingang (Signaleingang, Spannungsversorgung, Masse-Potenzial, etc.) einer Analogschaltung einkoppeln. Die zu untersuchende Schaltung wird dazu durch einen Wiener/Hammerstein-Ansatz als nichtlineares System mit zwei Eingängen modelliert. Mit Hilfe dieses Blockmodells werden analytische Ausdrücke derjenigen Frequenzanteile bestimmt, die durch Intermodulationen zwischen Stör- und Eingangssignal am Ausgang einer Schaltung entstehen.
\end{abstract}

\section{Einleitung}

Im Zuge der Entwicklung komplexer On-Chip-Systeme umfassen drahtlose Kommunikationssysteme immer größere Frequenzbereiche. Bei zeitgleicher Verringerung der Versorgungsspannungen steigen dadurch zunehmend die Anforderungen an elektrische Systeme in Bezug auf die elektromagnetische Zuverlässigkeit (engl.: Electromagnetic Reliability, kurz EMR) (Ramdani et al., 2009, 2008). Die Gewährleistung der Immunität von Sensor- und Sende/Empfangsschaltungen gegenüber elektromagnetischen Interferenzen, die in der Einsatzumgebung dieser Systeme auftreten, ist von grundlegender Bedeutung. Zur Sicherstellung eines zuverlässigen Betriebs müssen daher geeignete Modellansätze zur Untersuchung der Störfestigkeit in den Entwurfsprozess analoger Schaltungen einbezogen werden.

Unter Einfluss von elektromagnetischen Interferenzen (EMI) kann das Ausgangssignal einer Schaltung Störanteile enthalten, die von den einkoppelnden Störsignalen herrühren. Dadurch kann die Funktion einer elektrischen Schaltung bezüglich der Signalübertragung beeinträchtigt werden. In dieser Arbeit werden ausschließlich leitungsgefährte Störungen betrachtet, so dass die Störungen als zusätzliche Eingangssignale nach Abb. 1 aufgefasst werden können. Diese werden durch Quellen nachgebildet, die das Störsignal an einem beliebigen Eingang (Nomineller Signaleingang, Spannungsversorgung, Masse-Potenzial, etc.) darstellen. Aufgrund der Kennlinien nichtlinearer Bauelemente werden am Ausgang Frequenzanteile als Intermodulationen zwischen Signal und Störung entstehen. Im Folgenden wird die zu untersuchende Schaltung gemäß eines Wiener/Hammerstein-Modells mit mehreren Eingängen in lineare und nichtlineare Blöcke zerlegt. Mit Hilfe dieses Modells können die Einflüsse von Signal und Störung untersucht und analytische Ausdrücke der am Ausgang entstehenden Frequenzanteile bestimmt werden. Am Beispiel einer Differenz-Eingangsstufe wird dessen Verhalten bei Störungen auf der Versorgungsleitung untersucht.

\section{EMI-Blockmodell zu Untersuchung der Störbeeinflussung}

Aufgrund der nichtlinearen Übertragungscharakteristiken der Bauelemente einer Schaltung werden außer der Nutzfrequenz am Ausgang einer Schaltung durch StörEinflüsse weitere Spektralanteile auftreten. Diese Spektralanteile können sowohl harmonische Verzerrungen des Nutz- und des Störsignals (Frequenzvervielfachung) als auch Intermodulationen (Mischprodukte) dieser Eingangsanregungen sein. Diejenigen Effekte, welche die Signalübertragung eines Schaltungsblockes unter Störeinfluss am stärksten beeinflussen, sind die entstehenden Intermodulationsprodukte am Ausgang, welche innerhalb des Nutzbandes auftreten können. Da durch Intermodulationen zwischen Signal und Störung auch Signalanteile bei der Frequenz $f=0 \mathrm{~Hz}$ entstehen können (Wambacq et al. , 1998; Weiner and Spina, 1980), können aufgrund der einkoppelnden Störung auch die DC-Arbeitspunkte des Ausgangs und der Nachfolgenden Schaltungsblocke verschoben werden (sog. DC-Shift, vgl. Redouté and Steyaert , 2010b; Loeckx, 2010). Bei der Untersuchung der Einflüsse von Störungen auf die Funktion einer Schaltung werden in 


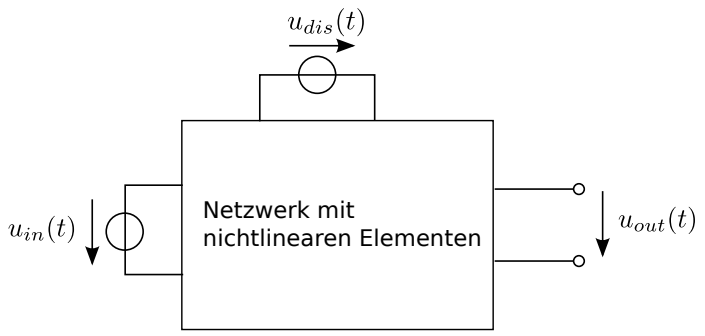

Abb. 1. Störeinfluss auf eine Schaltung mit nichtlinearen Elementen.

der Literatur dabei zumeist Störungen betrachtet, welche am nominellen Signaleingang einkoppeln (für Eingangsstufen s. z.B. Fiori, 2002; Redouté and Steyaert, 2010a). Um die Frequenzabhängigkeit des Einflusses einer Störung an einem Nicht-Signaleingang auf eine nichtlineare Schaltung zu beschreiben, kann der Störpfad als lineare Übertragungsfunktion beschrieben werden. Beispielhaft soll die Differenzstufe nach Abb. 2 bei Störung der Spannungsversorgung $U_{\mathrm{DD}}$ betrachtet werden. Eine Störung $u_{\text {dis }}(t)$, die der konstanten Versorgungsspannung überlagert ist, wird über den als Diode geschalteten Transistor $T_{5}$ sowie über den Stromspiegel $T_{3}$ und $T_{4}$ den Bias-Strom $i_{0}(t)$ des Differenz-Paares $T_{1}$ und $T_{2}$ verändern. Das Differenzpaar, bestehend aus den Transistoren $T_{1}$ und $T_{2}$, bildet die nichtlineare Übertragungsfunktion der Gesamtschaltung. Der frequenzabhängige (dynamische) Einfluss von $u_{\text {dis }}(t)$ auf diese Nichtlinearität kann beschrieben werden durch die Übertragungsfunktion $H_{\text {dis }}\left(f_{\text {dis }}\right)$ :

$H_{\text {dis }}\left(f_{\text {dis }}\right)=\frac{U_{\mathrm{gs}, 3}\left(f_{\text {dis }}\right)}{U_{\text {dis }}\left(f_{\text {dis }}\right)}$

Aufgrund von Störungen der Versorgungsleitungen wird die Gate-Source-Spannung $u_{\mathrm{gs}, 3}(t)$ von $T_{3}$ und damit der Strom $i_{0}(t)$ zeitabhängig. Die Ausgangsströme $i_{1}(t)$ und $i_{2}(t)$ werden aufgrund der nichtlinearen Übertragungscharakteristik Signalanteile von $u_{\text {dis }}(t)$ enthalten (vgl. Prinzip des SingleBalanced-Mixer).

Für eine vollständige Beschreibung aller Signalanteile, muss eine entsprechende Übertragungsfunktion $H_{\text {in }}$ auch für den Signalpfad aufgestellt werden. Die zu untersuchende Schaltung wird demnach in lineare, dynamische Blöcke und einen nichtlinearen Block mit zwei Eingängen aufgetrennt (s. Abb. 3). Die beiden linearen Blöcke sind dabei durch ihre Übertragungsfunktionen $H_{\text {in }}\left(f_{\text {in }}\right)$ und $H_{\text {dis }}\left(f_{\text {dis }}\right)$ vollständig beschrieben. Die Zwischengrößen $\tilde{x}_{\text {in }}$ und $\tilde{x}_{\text {dis }}$ für die gefilterten Eingangssignale $x_{\mathrm{in}}(t)=\hat{X}_{\text {in }} \cos \left(2 \pi f_{\text {in }} t\right)$ und $x_{\text {dis }}(t)=\hat{X}_{\text {dis }} \cos \left(2 \pi f_{\text {dis }} t\right)$ ergeben sich zu:

$\tilde{x}_{\text {in }}(t)=\hat{X}_{\text {in }} H_{\text {in }}\left(f_{\text {in }}\right) \cos \left(2 \pi f_{\text {in }} t\right)$,

$\tilde{x}_{\mathrm{dis}}(t)=\hat{X}_{\mathrm{dis}} H_{\mathrm{dis}}\left(f_{\mathrm{dis}}\right) \cos \left(2 \pi f_{\mathrm{dis}} t\right)$

Der Block $H_{\text {in }}$ stellt den Frequenzgang des Signalpfades und $H_{\text {dis }}$ den Frequenzgang des Störpfades dar (Stegemann,

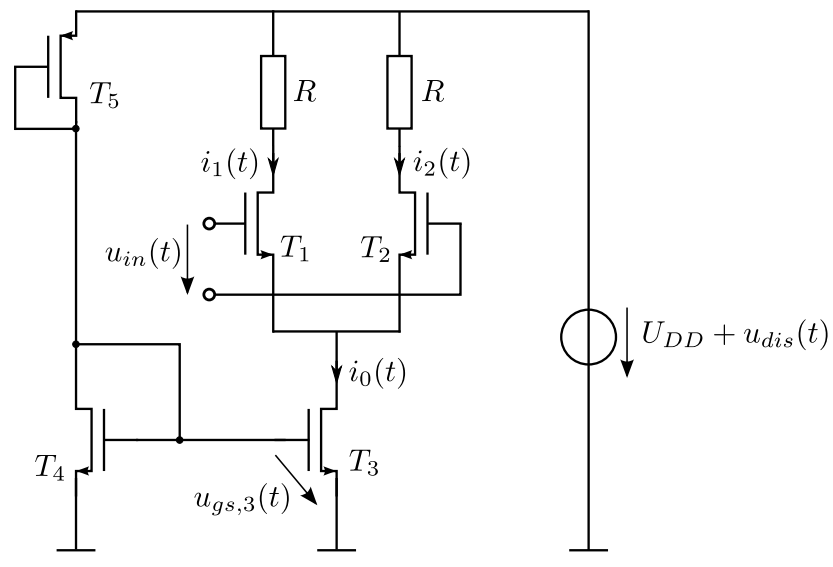

Abb. 2. Differenz-Eingangsstufe.

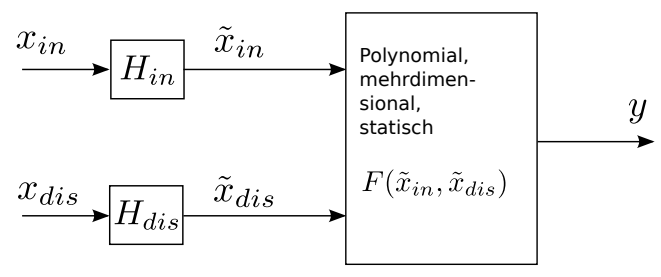

Abb. 3. Blockmodell zur Störuntersuchung.

2011). Auf diese Weise ist es möglich, die Unterschiedlichen Übertragungscharakteristiken von Stör- und Eingangssignal zu berücksichtigen. Die zu untersuchunde Schaltung ist also in demjenigen Frequenzbereich am Störanfälligsten, in dem $H_{\text {dis }}\left(f_{\text {dis }}\right)$ die größte Verstärkung aufweist.

Im Folgenden soll der nichtlineare Block keine weitere Dynamik enthalten, so dass seine statische, zweidimensionale Kennlinie durch das folgende multivariates Polynom beschrieben werden kann:

$y(t)=\sum_{i=0}^{N} \sum_{j=0}^{N-i} a_{i j} \tilde{x}_{\mathrm{in}}^{i}(t) \tilde{x}_{\mathrm{dis}}^{j}(t)$

Hierbei $a_{i j}$ die sog. Nichtlinearitätskoeffizienten (vgl. Wambacq et al. , 1998). Die Summen in Gl. (4) können wie folgt umgeschrieben werden:

$$
\begin{aligned}
y(t) & =a_{00}+\sum_{i=0}^{N} a_{j 0} \tilde{x}_{\mathrm{in}}^{j}(t)+\sum_{j=0}^{N} a_{0 j} \tilde{x}_{\mathrm{dis}}^{j}(t)+ \\
& +\sum_{k=1}^{N} \sum_{l=1}^{N} a_{k l} \tilde{x}_{\mathrm{in}}^{k}(t) \tilde{x}_{\mathrm{dis}}^{l}(t)
\end{aligned}
$$

Die ersten beiden Summen in Gl. (5) bestimmen ausschließlich Mischprodukte der an den Eingängen $\tilde{x}_{\text {in }}$ und $\tilde{x}_{\text {dis }}$ anliegenden Frequenzanteile. Die Intermodulationen zwischen Signal und Störung werden durch die letzte Summe 


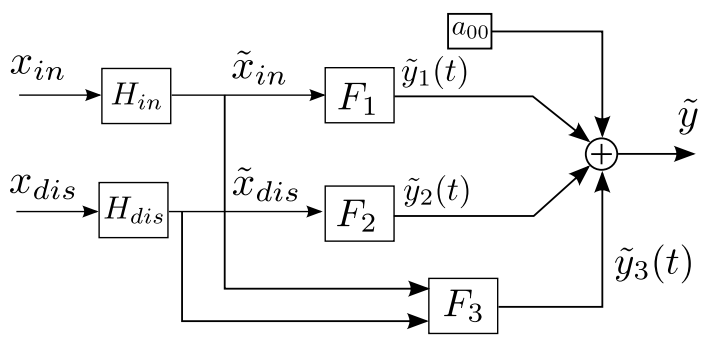

Abb. 4. Blockmodell: Aufteilung der multivariaten Nichtlinearität.

bestimmt. Mit Hilfe von Gl. (5) ergibt sich das äquivalente Blockmodell nach Abb. 4. Mit Hilfe dieser Blockstruktur können Einflüsse von Signal und Störung bezüglich der entstehenden Frequenzanteile im Ausgangssignal $y(t)$ getrennt untersucht werden. Durch Nullsetzen einer der beiden Eingänge $\tilde{x}_{\text {in }}$ oder $\tilde{x}_{\text {dis }}$ lässt sich das Modell in Abb. 4 auf ein klassisches Wiener/Hammerstein-Modell zurückführen, welches aus der Reihenschaltung eines linearen, dynamischen Blockes und eines statischen nichtlinearen Blockes besteht.

Die Störuntersuchung nichtlinearer Analogschaltungen mit Hilfe des in Abb. 3 dargestellten Modells wird nun wie folgt durchgeführt. Zunächst werden die Übertragungsfunktionen $H_{\text {in }}$ und $H_{\text {dis }}$ bestimmt. Diese können z.B. durch eine Kleinsignalanalyse für die im Arbeitspunkt linearisierte Schaltung für den Stör- bzw. den Signalpfad berechnet werden. Anhand des Frequenzganges des Störsignals wird derjenige Frequenzbereich identifiziert, in dem die Störungen die Signalübertragung der Schaltung am stärksten beeinflussen.

Im zweiten Schritt wird die multivariate Funktion $F\left(\tilde{x}_{\text {in }}, \tilde{x}_{\text {dis }}\right)$ des nichtlinearen Blockes bestimmt. Im folgenden Abschnitt wird diese Funktion mit Hilfe des Verfahrens der nichtlinearen Quellen bestimmt. Bei diesem Verfahren werden die Ströme und Spannungen gemäß der Annahmen zur Volterra-Analyse als additive Überlagerung der nichtlinearen Antworten verschiedener Ordnungen berechnet (s. z.B. (Maas, 2003), Kapitel 4). Mit Hilfe der Funktion $F\left(\tilde{x}_{\text {in }}, \tilde{x}_{\text {dis }}\right)$ können für Eingangssignale $x_{\text {in }}$ und $x_{\text {dis }}$, die sich als Linearkombination von Sinus- und Kosinusfunktionen darstellen lassen, die Ausgangsamplituden an denjenigen Frequenzen bestimmt werden, die sich durch Intermodulationen zwischen Eingangs- und Störsignal ergeben.

\subsection{Charakterisierung der Nichtlinearität}

Da das dynamische Verhalten des Gesamtsystems nach Abb. 3 durch die Übertragungsfunktionen der linearen Blöcke $H_{\text {in }}$ und $H_{\text {dis }}$ beschrieben sein soll, werden für die Berechnung des multivariablen Übertragungsverhaltens des nichtlinearen Blockes ausschließlich statische Elemente berücksichtigt. D.h. es werden nur lineare und nichtlineare Elemente mit Strom-Spannungsrelationen in Leitwertform $i=f_{g}(u)$ und in Widerstandform $u=f_{r}(i)$ berücksichtigt,

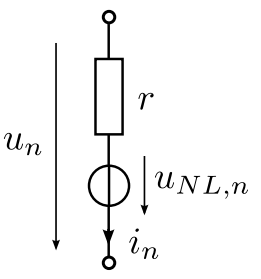

(a)

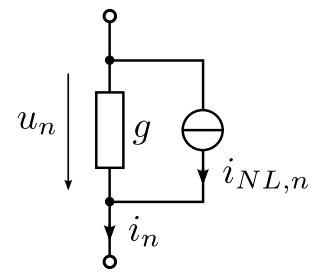

(b)
Abb. 5. Multilineares Modell nichtlinearer Netzwerkelemente. (a) Widerstandform und (b) Leitwertform.

die keine Abhängigkeiten von den zeitlichen Ableitungen der Strom- oder Spannungsverläufe aufweisen. Die Elemente in Leitwertform können Leitwerte oder gesteuerte Stromquellen sein. Die Elemente in Widerstandform können Widerstände, oder gesteuerte Spannungsquellen sein.

Entsprechend der Beschreibung durch Volterra-Reihen kann jede Knotenpsannung $u$ und jeder Zweigstrom $i$ eines nichtlinearen Netzwerkes durch Überlagerung der Systemantworten der Ordnung $n=1 \ldots N$ approximiert werden (Schetzen, 1985). Für die Ströme und Spannungen eines Netzwerkes mit nichtlinearen Elementen gilt dann:

$i(t) \approx \sum_{n=1}^{N} i_{n}(t)$

$u(t) \approx \sum_{n=1}^{N} u_{n}(t)$

Die Antworten $i_{n}(t)$ und $u_{n}(t)$ enthalten dabei ausschließlich Mischfrequenzen der Ordnung $n$ (vgl. z.B. Weiner and Spina, 1980).

Um das Ein-/Ausgangsverhalten einer nichtlinearen Schaltung zu beschreiben, werden die oben genannten nichtlinearen Elemente bei diesem Ansatz ersetzt durch lineare Elemente mit in Reihe, bzw. parallel geschalteten Quellen (Abb. 5). Die folgenden Berechnungen werden anhand eines nichtlinearen Elementes in Leitwertform dargestellt. Für nichtlineare Elemente in Widerstandform verlaufen die Berechnungen analog. Dabei wird angenommen, dass für die nichtlinearen Strom-Spannungsrelationen $f_{g}(u)$ (und $f_{r}(i)$ ) analytische Ausdrücke vorliegen, so dass sie in Potenzreihen der Form:

$i(t)=\sum_{m=1}^{N} k_{m} u^{m}(t)$,

als Aussteuerungen um einen Arbeitspunkt entwickelt werden können.

Aufgrund der multilinearen Eigenschaften von VolterraOperatoren führt die Skalierung jeder Quellengröße um 
einen konstanten Faktor $c$ in Gln. (6) und (7) zu einer Skalierung jeder Antwort der Ordnung $n$ mit $c^{n}$ (Schetzen, 1985):

$i(t)=\sum_{n=1}^{N} c^{n} i_{n}(t)$

$u(t)=\sum_{n=1}^{N} c^{n} u_{n}(t)$

Zusammen mit Gl. (8) ergibt sich für die Ströme der nichtlinearen Elemente:

$i(t)=\sum_{n=1}^{N} c^{n} i_{n}(t)=$

$\sum_{m=1}^{N} k_{m} u^{m}(t)=\sum_{m=1}^{N} k_{m}\left(\sum_{n=1}^{N} c^{n} u_{n}(t)\right)^{m}$.

In (Schetzen, 1985) wurde gezeigt, dass die Antworten für jedes $n$ bestimmt werden können, wenn die Summen in Gl. (11) ausgeschrieben und nach Potenzen von $c$ sortiert werden. Die Antworten der Knotenspannungen und Zweigströme der Ordnung $n$ eines nichtlinearen Elements können dann ausgedrückt werden durch die Überlagerung einer linearen Antwort und einer nichtlinearen $f_{\mathrm{NL}, n}$ Antwort:

$i_{n}(t)=g \cdot u_{n}(t)+f_{\mathrm{NL}, n}\left(u_{1}, \ldots, u_{n-1}\right)$.

Eine Stromkomponente $n$-ter Ordnung kann nach Gl. (12) durch einen linearen Leitwert mit dem Wert $g=k_{1}$ modelliert werden, an dem die Spannung $u_{n}(t)$ anliegt. Parallel zu diesem linearen Leitwert ist eine Stromquelle $n$-ter Ordnung mit $i_{\mathrm{NL}, n}=f_{\mathrm{NL}, n}\left(u_{1}, \ldots, u_{n-1}\right)$ geschaltet. Der Wert dieser Stromquelle hängt dabei nur von den Antworten der Ordnungen kleiner als $n$ ab. In Abb. 5 (b) ist die Modellierung nichtlinearer Leitwerte mit Hilfe der nichtlinearen Stromquellen $i_{\mathrm{NL}, n}(t)$ dargestellt. Nichtlineare Elemente in Widerstandform werden durch in Reihe geschaltete Spannungsquellen modelliert (s. Abb. 5a). Mit den Gln. (11) und (12) ergeben sich die folgenden Ausdrücke für die nichtlinearen Stromquellen der Ordnungen $n=1 \ldots 4$ :

$i_{\mathrm{NL}, 1}=0$

$i_{\mathrm{NL}, 2}=k_{2} u_{1}^{2}$

$i_{\mathrm{NL}, 3}=2 k_{2} u_{1} u_{2}+k_{2} u_{1}^{3}$

$i_{\mathrm{NL}, 4}=k_{2}\left(2 u_{1} u_{3}+u_{2}^{2}\right)+3 k_{3} u_{1}^{2} u_{2}+k_{4} u_{1}^{4}$

Die hier verwendete Methode bietet den Vorteil, dass zur Berechnung der Antworten $n$-ter Ordnung ausschließlich lineare Netzwerke mit linearen Elementen berechnet werden müssen. Das nichtlineare Verhalten der Schaltung wird durch das Hinzufügen von nichtlinearen Quellen $n$-ter Ordnung modelliert (vgl. Abb. 6). Intermodulationsprodukte und harmonische Verzerrungen $n$-ter Ordnung werden dabei ausschließlich durch die Quellen $u_{\mathrm{NL}, n}$ und $i_{\mathrm{NL}, n}$ gebildet.

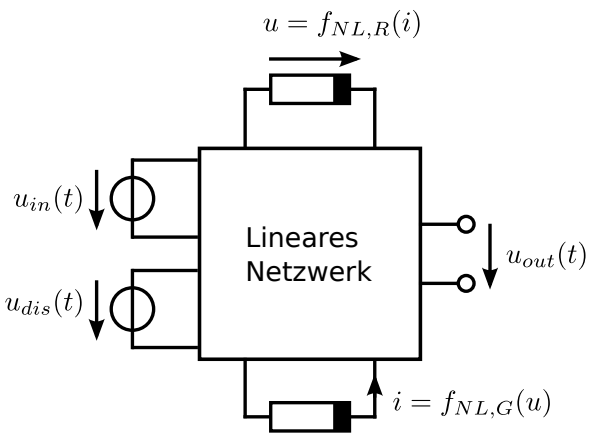

(a)

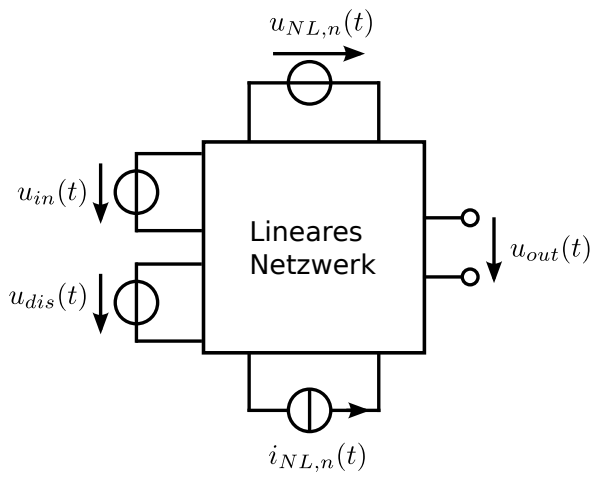

(b)

Abb. 6. (a) Netzwerk mit nichtlinearen Elementen. (b) Linearisiertes Ersatznetzwerk mit nichtlinearen Quellen $n$-ter Ordnung.

Die Berechnung der nichtlinearen, statischen Kennlinie $y=F\left(\tilde{x}_{\text {in }}, \tilde{x}_{\text {dis }}\right)$ zur Störuntersuchung eines Netzwerkes läuft dabei gemäß der Methode der nichtlinearen Quellen ab wie folgt:

- Die nichtlinearen Strom-Spannungsrelationen der nichtlinearen Netzwerkelemente werden in eine Potenzreihe um den Arbeitspunkt entwickelt (Taylorreihe). Alle nichtlinearen Netzwerkelemente werden durch ihren linearisierten Leitwert bzw. Widerstand ersetzt (erster Taylorkoeffizient).

- Antwort erster Ordnung: Die Ausgangsspannung(der Ausgangsstrom) sowie alle Steuergrößen der nichtlinearen Elemente des linearisierten Netzwerkes werden für die Eingangsanregung bestimmt.

- Antworten höherer Ordnung $(N>n>1)$ : die Eingangsquellen werden "genullt". Entsprechend den Gln. (13) bis (16) werden die nichtlinearen Quellen mit der Antwort der Ordnung $(n-1)$ berechnet und gemäß 5 in das Netzwerk eingefügt. Die Ausgangsspannung (der Ausgangsstrom) sowie alle Steuerspannungen der Ordnung $n$ werden für die Anregung mit den nichtlinearen Quellen $n$-ter Ordnung bestimmt. 


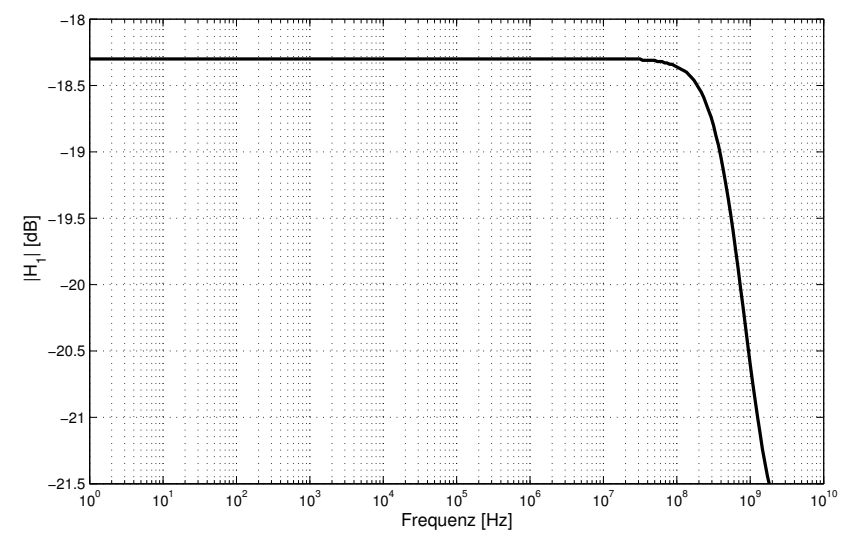

Abb. 7. Lineare Übertragungsfunktion $H_{\text {dis }}$ des Differenzverstärkers für Störungen an $U_{\mathrm{DD}}$.

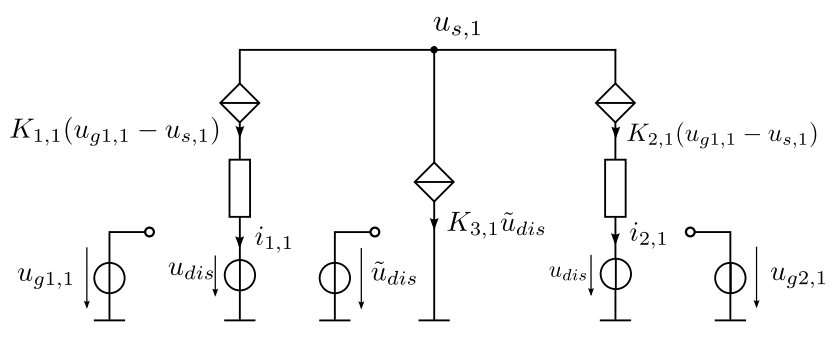

Abb. 8. Netzwerk zur Berechnung der Antworten erster Ordnung.

Durch die Überlagerungen der einzelnen Antworten ergibt sich die Approximation der Zeitverläufe der Ausgangsspannung bzw. des Ausgangsstroms in Abhängigkeit der Eingangsquellen. Aufgrund der Entwicklung der einzelnen Nichtlinearitäten in eine polynomielle Form nach Gl. (8) ergibt sich für die Ein-/Ausgangsbeschreibung ebenfalls eine polynomielle Darstellung (Stegemann, 2011).

\section{Beispiel: Differenz-Eingangsstufe}

Im Folgenden soll die Störanalyse der Differenz-Eingangsstufe in Abb. 2 für Störungen $u_{\text {dis }}=\hat{U}_{\text {dis }} \cos \left(2 \pi f_{\text {dis }} t\right)$ an der Spannungsversorgung untersucht werden. Die Aufteilung in die Blöcke nach der in Abb. 3 dargestellten Struktur geschieht wie folgt. Die Übertragungsfunktion $H_{\text {dis }}$ ist gegeben durch Gl. (1) und repräsentiert den Einkopplungspfad des Störsignals über $T_{5}$ und den Stromspiegel $T_{3} / T_{4}$. Der durch eine Kleinsignalanalyse bestimmte Verlauf von $H_{\text {dis }}$ ist in Abb. 7 dargestellt. Dieser Block besitzt Tiefpasscharakteristik mit einer Grenzfrequenz von $\sim 2 \mathrm{GHz}$. Es ist zu erwarten, dass die Differenzstufe im Durchlassbereich von $H_{\text {dis }}$ am Störempfindlichsten ist. Der lineare Block $H_{\text {sig }}$ repräsentiert das dynamische Verhalten der Eingangsspannung $u_{\text {in }}(t)$ auf den Ausgangsstrom $i_{\text {out }}(t)=i_{1}(t)-i_{2}(t)$. Das

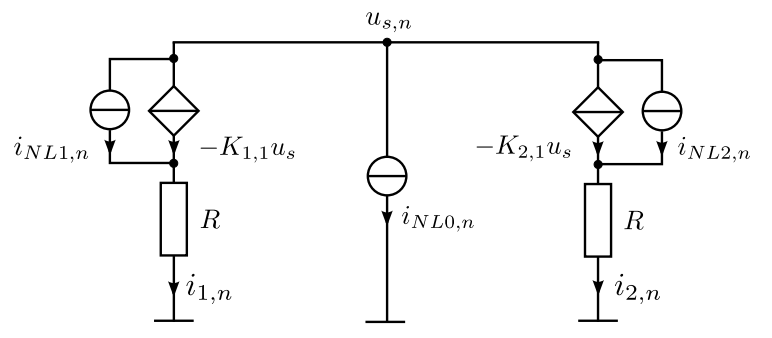

Abb. 9. Netzwerk zur Berechnung der Antworten $n$-ter Ordnung.

Frequenzverhalten wird dominiert durch die Gate-SourceKapazitäten der Transistoren $T_{1}$ und $T_{2}$. Auch dieser Block weist Tiefpasscharakteristik auf. Aufgrund des Frequenzverhaltens beider Blöcke können zum einen Störfrequenzen $f_{\text {dis }}$ in der Nähe der Signalfrequenzen $f_{\text {sig }}$ Intermodulationen im Nutzband der Differenzstufe erzeugen. Zum anderen können aufgrund der Bandbreite des Blockes $H_{\text {dis }}$ auch Intermodulationen zwischen zwei Störfrequenzen $f_{\text {dis1 }}$ und $f_{\text {dis2 }}$, mit $f_{\text {dis1 }}>f_{\text {dis2 }}$ aus dem Durchlassbereich von $H_{\text {dis }}$ in das Nutzband fallen, wenn die Differenz $f_{\text {dis } 1}-f_{\text {dis } 2}$ kleiner ist, als die Bandbreite des Blockes $H_{\text {in }}$. Für eine Störuntersuchung bieten sich daher die Intermodulationsprodukte zweiter und dritter Ordnung an (Stegemann, 2011). Die Abbn. 8 und 9 zeigen die Ersatznetzwerke zur Berechnung der nichtlinearen Charakteristik nach Gl. (5) mit dem in Kapitel 2.1 beschriebenen Verfahren. Die Transistoren $T_{1}, T_{2}$ und $T_{3}$ sind durch Spannungsgesteuerte Stromquellen ersetzt worden, deren Kennlinie um den Arbeitspunkt in eine Taylorreihe entwickelt wurde. Dabei seien $k_{1, i}=k_{2, i}, i=1 \ldots N$, die $i$-ten Nichtlinearitätskoeffizienten von $T_{1}$ und $T_{2} . k_{3, i}$ seien die Nichtlinearitätskoeffizienten von $T_{3}$.

Der differenzielle Ausgangsstrom $i_{\text {out }}$ ergibt sich für Gegentaktaussteuerung mit $u_{g 1,1}=-u_{g 2,1}=0,5 u_{\text {in }}=$ $\hat{U}_{\text {in }} \cos \left(2 \pi f_{\text {in }} t\right) \mathrm{zu}$ :

$i_{\text {out }, 1}=i_{1,1}-i_{2,1}=2 k_{1,1} u_{\text {in }}$.

Die Stromkomponenten erster bis dritter Ordnung berechnen sich zu:

$$
\begin{aligned}
i_{\text {out }, 1} & =k_{1,1} \tilde{u}_{\text {in }}, \\
i_{\text {out }, 2} & =\frac{-k_{1,2} k_{3,1}}{k_{1,1}} \tilde{u}_{\text {in }} \tilde{u}_{\text {dis }}, \\
i_{\text {out }, 3} & =\left(\frac{3 k_{1,3} k_{3,1}^{2}}{4 k_{1,1}^{2}}-\frac{k_{1,2}^{2} k_{3,1}^{2}}{2 k_{1,1}^{3}}-\frac{k_{1,2} k_{3,2}}{k_{1,1}}\right) \tilde{u}_{\text {in }} \tilde{u}_{\text {dis }}^{2} \\
& +\left(\frac{-k_{1,2}^{2}}{2 k_{1,1}}+\frac{k_{1,3}}{4}\right) \tilde{u}_{\text {in }}^{3} .
\end{aligned}
$$

Gl. (19) beinhaltet Intermodulationen zweiter Ordnung zwischen Störung und Eingangssignal. Die Antwort dritter 


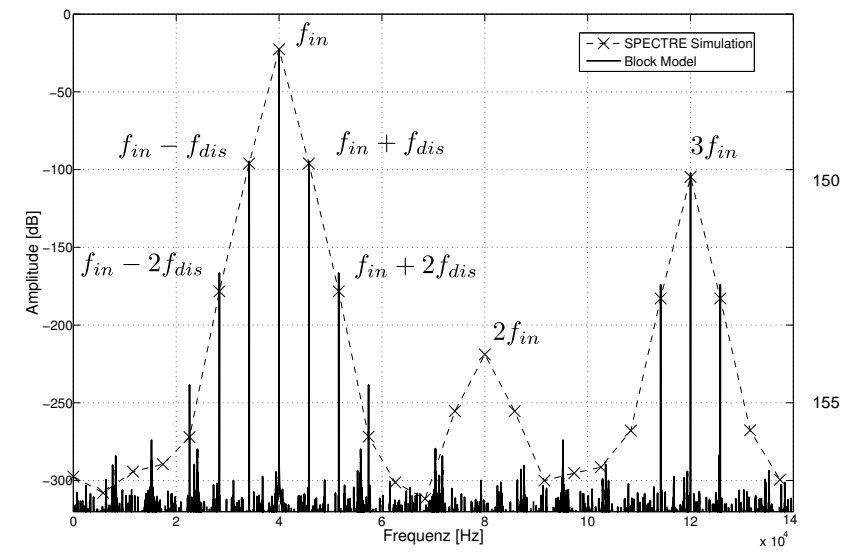

Abb. 10. Vergleich des Ausgangsspektrums mit Harmonic-BalanceSimulation des Differenzverstärkers.

Ordung aus Gl. (20) beinhaltet Intermodulationen dritter Ordnung, deren Amplituden durch den Vorfaktor von $\tilde{u}_{\text {in }} \tilde{u}_{\text {dis }}^{2}$ bestimmt sind. Die Amplituden an den Intermodulationsfrequenzen zweiter und dritter Ordnung, die sich aus den beiden Eingangssignalen $x_{\text {in }}(t)$ und $x_{\text {dis }}(t)$ ergeben, lassen sich mit Gln. (2) und (3) bestimmen zu (Stegemann, 2011):

$A_{2 \text { nd }}=k_{2}^{*} H_{\text {in }}\left(f_{\text {in }}\right) H_{\text {dis }}\left(f_{\text {dis }}\right) \hat{U}_{\text {in }} \hat{U}_{\text {dis }}$,

$A_{3 \mathrm{rd}}=k_{3}^{*} H_{\text {in }}\left(f_{\text {in }}\right) H_{\text {dis }}^{2}\left(f_{\text {dis }}\right) \hat{U}_{\text {in }} \hat{U}_{\text {dis }}^{2}$.

Wobei die folgenden Abkürzungen eingeführt wurden:

$k_{2}^{*}=\frac{-k_{1,2} k_{3,1}}{k_{1,1}}$

$k_{3}^{*}=\left(\frac{3 k_{1,3} k_{3,1}^{2}}{4 k_{1,1}^{2}}-\frac{k_{1,2}^{2} k_{3,1}^{2}}{2 k_{1,1}^{3}}-\frac{k_{1,2} k_{3,2}}{k_{1,1}}\right)$

In Abb. 10 sind die Ausgangsspektren des Block-Modells und das Ergebnis einer Harmonic-Balance-Simulation (HBS) für die untersuchte Differenzstufe gegenüber gestellt. Für das Störsignal galt in beiden Fällen $\hat{U}_{\text {dis }}=1 \mathrm{mV}$ und $f_{\text {dis }}=5,8 \mathrm{kHz}$ sowie für das Eingangssignal $\hat{U}_{\text {in }}=10 \mathrm{mV}$ und $f_{\text {in }}=40 \mathrm{kHz}$. Die Antworten zweiter und dritter Ordnungen liefern für die Intermopdulationsfrequenzen $f_{\text {in }} \pm$ $f_{\text {dis }}$ und $f_{\text {in }} \pm 2 f_{\text {dis }}$ gute Übereinstimmungen. Da im Blockmodell nur das Gegentaktverhalten angenommen wurde, treten im Gegensatz zur HBS keine Frequnezanteil bei $2 f_{\text {in }}$ auf.

\section{Zusammenfassung}

In diesem Beitrag wurde ein block-orientiertes Modell vorgestellt, mit welchem die Einflüsse von Störungen untersucht werden können, die auf einen beliebigen Nicht-Signaleingang einer Schaltung (Spannungsversorgung, Masse-Potenzial, etc.) einwirken. Mit dem hier verwendeten multi-input Wiener/Hammerstein-Ansatz wird die zu untersuchende Schaltung in lineare dynamische und nichtlineare statische Blöcke zerlegt, so dass die Einflüsse von Signal- und Störpfad bezüglich des linearen Frequenzverhaltens getrennt betrachtet werden können. Am Beispiel einer Differenz-Eingangsstufe wurden analytische Ausdrücke für die Amplituden an denjenigen Intermodulationsfrequenzen bestimmt, die sich durch Einkopplung von Störsignalen in die Versorgungsspannung ergeben.

Danksagung. Das diesem Beitrag zugrundeliegende Vorhaben BatMan wird mit Mitteln des Bundesministeriums für Bildung und Forschung (BMBF) unter dem Kennzeichen 03X4604E gefördert. Die Verantwortung für den Inhalt dieser Veröffentlichung liegt allein bei den Autoren.

\section{Literatur}

Fiori, F.: A new nonlinear model of EMI-induced distortion phenomena in feedback CMOS operational amplifiers, Transactions on Electromagnetic Compatibility, IEEE, 44, 495-502, 2002.

Loeckx, J.: Methods for simulating and analysing the effects of EMC on Integrated Circuits, Ph.D. thesis, Katholieke Universiteit Leuven, 2010.

Maas, S. A.: Nonlinear Microwave and RF Circuits, Artech House, 2nd Edn., 2003.

Ramdani, M., Sicard, E., Ben Dhia, S., and Catrysse, J.: Towards an EMC roadmap for Integrated Circuits, Asia-Pacific Symposium on Electromagnetic Compatibility and 19th International Zurich Symposium on Electromagnetic Compatibility, 2008

Ramdani, M., Sicard, E., Boyer, A., Ben Dhia, S., Whalen, J., Hubing, T., Coenen, M., and Wada, O.: The Electromagnetic Compatibility of Integrated Circuits - Past, Present, and Future, IEEE Transactions on Electromagnetic Compatibility, 51, 78-100, 2009.

Redouté, J.-M. and Steyaert, M.: EMI-Resistant CMOS Differential Input Stages, IEEE Transactions on Circuits and Systems I: Regular Papers, 57, 323-331, 2010.

Redouté, J.-M. and Steyaert, M.: EMC of Analog Integrated Circuits, Analog Circuits and Signal Processing, Springer, 2010.

Schetzen, M.: Multilinear Theory of Nonlinear Networks, Journal of the Franklin Institue, Volume 320, 221-247, 1985.

Stegemann, S., John, W., and Mathis, W.: Blockorientierte Analyse der Empfindlichkeit nichtlinearer Schaltungen gegenüber elektromagnetischen Störungen an Nicht- Signaleingängen, Proc. 12. GMM/ITG-Fachtagung ANALOG 2011, 2011.

Wambacq, P. and Sansen, W.: Distortion Analysis of Analog Integrated Circuits, Kluwer Academic Publishers, 1998.

Weiner, D. D. and Spina, J. F.: Sinusoidal Analysis and Modeling of weakly nonlinear Circuits, van Nostrand Reinhold, 1980. 